\title{
Development of ZIF-8 membranes: opportunities and challenges for commercial applications
}

\author{
Zhiping Lai
}

Metal organic framework (MOF) membranes have attracted significant attentions in recent years because of their potentials in gas and liquid separations and other applications such as catalysis and chemical sensors, etc. More than half of the MOF membrane publications up to date are related to ZIF-8 system, because of its easy synthesis, relatively high stability, and excellent gas separation performance, which allows many novel ideas to be easily implemented. Extensive studies have shown that ZIF-8 membranes hold great potentials in gas separations, but may face great challenges in liquid separations mainly because of their poor stability. This is also a common observation for other MOF membranes. As such, in this article we use ZIF-8 membrane as a prototype and focus on its development in gas separations for the discussions of the most concerned issues related to membrane commercialization including membrane synthesis, separation performance, stability, process reproducibility, and finally on the opportunities and challenges that MOF membranes may face in industrial applications.

\section{Addresses}

Advanced Membranes and Porous Materials Center, Physical Science and Engineering, King Abdullah University of Science and Technology, Thuwal, 23955, Saudi Arabia

Corresponding author: Lai, Zhiping (Zhiping.lai@kaust.edu.sa)

\section{Introduction}

Membrane technology offers great energy benefit in the separation of bulk gas and liquid mixtures since the separation can be carried out without phase separation. In an optimal membrane process design, the energy of the entire system is mainly determined by the 
membrane selectivity, so it should be the first important design parameter to consider. The higher the selectivity, the lower the energy consumption can be achieved $\left[1,2^{\circ}\right]$. To save membrane cost, the membrane permeance is the second important design parameter which should also be as high as possible. The third design parameter is pressure ratio between the feed and permeate in order to meet the product purity requirement. Hence, a membrane should be robust enough to hold a certain transmembrane pressure drop (TMP).

As a new type of ordered porous materials, MOFs hold great potentials to provide both high selectivity and high permeability because of their uniform pore size for sharp molecular sieving and high porosity for fast transport rate. ZIF-8 is a small pore MOF which consists of zinc ions coordinated with 2-methyl imidazolate ligands, forming a SOD zeolite structure with connection window size of $0.34 \mathrm{~nm}$ calculated from the crystallographic structure. ZIF-8 is first reported by Chen et al. [3*0] and Yaghi et al. [4]. ZIF-8 has many advantages: It is one of the most stable MOF structures [5]. The starting materials are cheap and easily available. The synthesis is relative easy. Wiebcke et al. first reported a fast and room temperature synthesis protocol using methanol as solvent $\left[6^{\circ}\right]$. We first reported the synthesis in aqueous solution $\left[7^{\circ *}\right]$. The first ZIF-8 membrane was reported by Caro $\left[8^{\bullet *}\right.$. The pore size is suitable to separate a number of important gas mixtures including $\mathrm{H}_{2} / \mathrm{CO}_{2}, \mathrm{CO}_{2} / \mathrm{N}_{2}, \mathrm{H}_{2} / \mathrm{CH}_{4}$, and most notably, $\mathrm{C}_{2} / \mathrm{C}_{3}$ and propylene/propane. However, there are also many challenges in the MOF membrane preparation and applications, such as brittleness, cracks, reproducibility, process scalability, etc. These challenges have significantly slowed down the commercialization roadmap of the counterpart zeolite membranes. In addition, MOFs are mechanically softer and chemically weaker compared to zeolites. All of these challenges and weaknesses have cast a big concern to the future of MOF membranes [911]. Nevertheless, the fascinating MOF structures have attracted more and more talent researchers into the field and many novel ideas have been proposed to tackle these challenges in 
recent years. Hence, it is worthy to revisit this topic, especially from the practical application point of view.

\section{Membrane synthesis}

Like other crystalline membrane materials such as zeolites, the membrane performance is related to many factors such as pore size, structure flexibility, orientation, and thickness. Since all MOF membranes developed thus far are polycrystalline, hence grain boundaries and pinholes are important membrane microstructures that if not properly sealed can be a major source of defects. It is worthy to note that the maximum size difference between any gas molecules is less than 0.3 $\mathrm{nm}$, which is beyond the resolution of most SEM and TEM equipment. Hence, even a membrane appears compact under SEM or TEM inspection, the membrane could still fail to offer good separation performance. Hence, gas separation membranes are particularly sensitive to defects, and this has imposed a big challenge to membrane preparation. Although many novel membrane fabrication methods have been developed which can be found in details in a number of review articles $\left[12-13,14^{\bullet \bullet}, 15\right]$, only the in situ crystallization, seeded growth, and counter-diffusion (CD) methods, which are illustrated in Figure 1, have yielded membranes with decent gas separation performance.

The normal in situ crystallization method is simple but suffers from poor heterogeneous nucleation on the surface of supports. Many methods including surface modification of the support with metal coordination agents [16-18], coating a ZnO layer [19-22] or using polymer with metal chelating groups [23], are proposed to enrich the metal ions on the support. These methods improve not only the process reproducibility, but also the adhesion between the membrane and support. 
The seeded growth method uses a seed layer to by-pass the nucleation stage and promote growth on the support surface. This method is able to control membrane orientation, thickness and grain boundary structure, and hence often leads to a better membrane performance [24]. The seed layer is typically prepared by dip-coating or rubbing $[25,26]$, but both methods suffer from nonuniformity. Jeong et al. developed a microwave assistant seeding technique in which a uniform seed layer can be formed in situ on the support surface. After seeded growth, high quality ZIF-8 membranes with good propylene/propane separation performance are achieved [27].
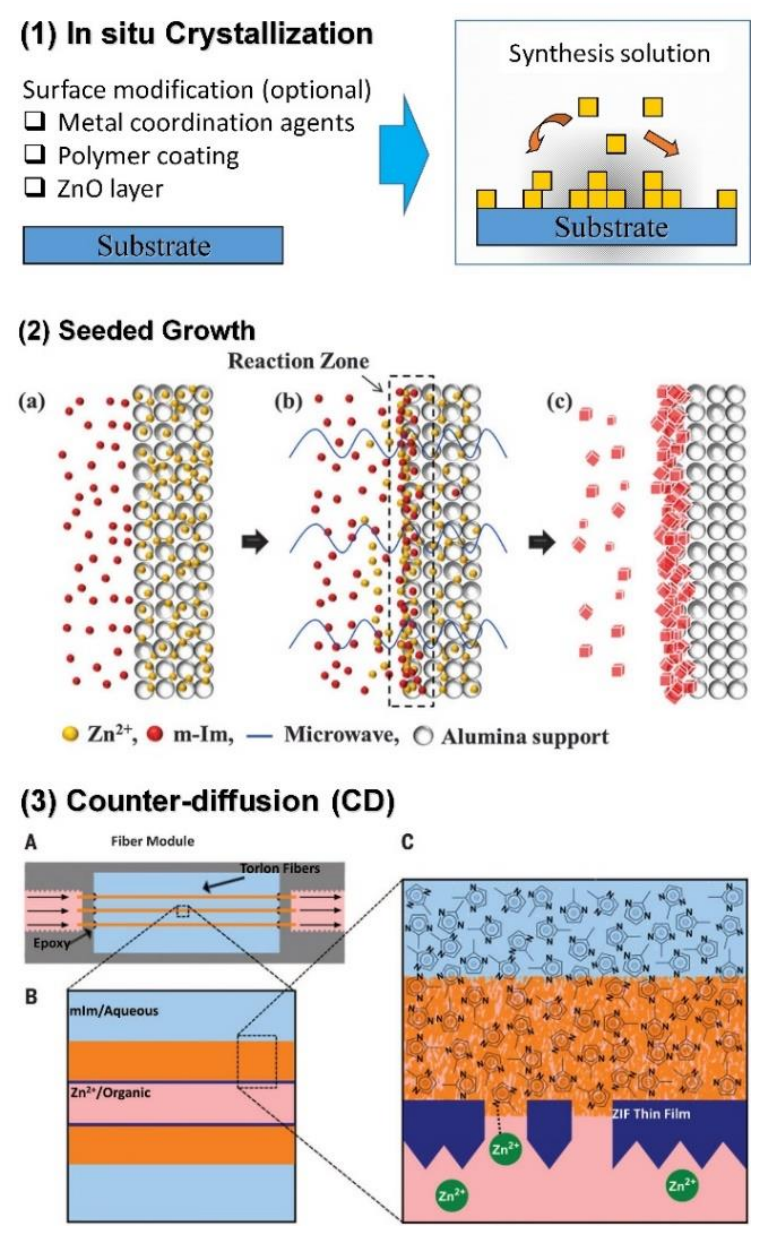

Figure 1: Illustrations of the three most effective ZIF-8 membrane fabrication methods. (1) in situ crystallization; (2) a microwave assisted seeded growth process [27]; (3) an interfacial microfluidic counter-diffusion method [28*0]. 
The CD method was first developed by Wang et al.[29*0] utilizing the unique coordination chemistry of MOFs which are typically formed from two precursors, one is the metal salt which is soluble in either water or organic solvents, and the other is the organic ligand which is in most cases soluble only in organic solvent. 2-methylimidazole is one of the few imidazoles that are soluble in water, and hence ZIF-8 can be synthesized in pure aqueous solution [ $7^{\circ \bullet}$. A number of good progresses have been made in this method. Jeong et al. developed a two-step CD process in which the support is first soaked in one precursor solution and then immersed in another precursor solution[30*0. Nair et al. developed an interfacial microfluidic flow processing method in which the precursor solutions are supplied separately from two sides of the hollow fiber support. The membrane can be formed at any position of the support $\left[28^{\circ}, 31\right]$. A number of other studies also reported the flow process where the precursor solutions are cycled either from one side or both sides of the support [32-34]. Cycling the precursor solution can promote not only the product yield, but also the crystallization rate $\left[28^{\bullet \bullet}, 31\right]$. In-situ TEM observation on ZIF-8 crystal growth confirmed that the crystallization rate is much faster in a flow system than that in a static solution [35].

The support is also an important factor that will affect the membrane quality. The surface chemistry, roughness, and mechanical strength of the support sometimes will greatly affect the membrane growth, the adhesion, and the integrity of the final membrane. Commonly used supports include ceramics and polymers. In most cases the support should be inert since the support should be robust and stable in practical applications. However, inert supports typically have poor heterogeneous nucleation and weak adhesion. This is particular an issue in the in situ crystallization method as discussed previously. While in the seeded growth method, the effect of the support can be largely eliminated by the seed layer. Supports with a certain degree of roughness can enhance the adhesion, but a smooth surface is often needed to achieve a more 
uniformly oriented and thinner membrane. Ceramic supports have much stronger mechanical strength than polymer supports, so presumably ceramic supports should be beneficial for the polycrystalline ZIF-8 membranes, which is brittle and fragile in nature. However, a number of reports have showed that under low TMP conditions polymer supported ZIF-8 membranes are able to achieve similar gas separation performances as those on ceramic supports $\left[23,28^{\bullet \bullet}, 31\right]$. While under high TMP the membrane performance in both cases is unknown and need study more.

Compared to zeolite membranes, ZIF-8 system has many advantages towards commercialization, as summarized below.

- The synthesis can be carried out at room temperature, or at temperature below the boiling point of the solvent used. This eliminates the use of expensive autoclaves and makes the process much easier to handle.

- The synthesis time is short. The synthesis time can be further reduced by coupling with microwave heating [ $8^{\bullet *}$ or rapid thermal deposition [36].

- Since MOF syntheses do not require organic structure directing agents, there is no need for thermal sintering which is one of the major steps to form defects in zeolite membranes. The mild synthetic conditions allow the use of polymers as supports $\left[23,29^{\bullet \bullet}, 31,37^{\bullet}, 38^{-}\right.$ $\left.43,44^{\circ}\right]$, which could significantly reduce the membrane cost.

- The counter-diffusion method can repair defects in situ [30॰0].

\section{Gas separation performance}

The pore size of ZIF- 8 based on the crystallographic structure data is around $0.34 \mathrm{~nm}$, which is similar to the kinetic diameter of $\mathrm{CO}_{2}$. However, the gas permeation data exhibited only moderate selectivities for hydrogen and $\mathrm{CO}_{2}$ over other gases. For example, the selectivities of $\mathrm{H}_{2} / \mathrm{CO}_{2}, \mathrm{H}_{2} / \mathrm{N}_{2}$ 
and $\mathrm{H}_{2} / \mathrm{CH}_{4}$ are typically around 4,9 , and 13 , respectively. Such low selectivities will be difficult to compete with polymeric membranes and hence the potential of ZIF-8 membranes in these gas separations will be limited. A sharp molecular sieving is found between ethane/propane and propylene/propane $\left[45^{\bullet}, 46^{\bullet \bullet}\right]$, which indicates the effective pore size of ZIF-8 is around $0.42 \mathrm{~nm}$. This discrepancy in pore size is typically attributed to the flopping motion of the methyl group attached to the imidazole. However, even for zeolites whose structure is often considered rigid, the effective pore size is also found approx. $0.05 \mathrm{~nm}$ larger than the crystallographic pore size [47]. Detailed adsorption studies of ZIF-8 showed that the adsorption isotherm should be modelled by two structures: one is obtained at normal condition and the other at high pressure [48]. Change between these two structures is occurred at a relative adsorption pressure of $2 \times 10^{-4}$. Most of the reported permeation data are based on single-component and hence the reported selectivities are ideal selectivities. For many porous materials the mixture selectivities can be very different from the ideal ones due to effects such as single-file diffusion or selective adsorption, etc. Fortunately, for ZIF-8 membranes there is not much difference [49]. The reason is probably because that the ZIF-8 structure is composed of large cages connected with short channels, so even the single file effect or preferable adsorption occurs in the short channels, the molecules will redistribute inside the large cages.

The efficient separation of propylene and propane over ZIF-8 membranes is an exciting discovery

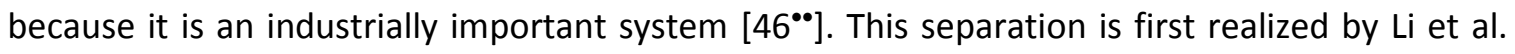
from the kinetic adsorption studies over chlorated-ZIF-8 powder [50]. The size difference between these two molecules is around $0.02 \mathrm{~nm}$, while the reported membrane selectivity is as high up to 150 [23]. The separation is apparently based on the kinetic difference because their adsorption capacities are almost the same. Hence, this system clearly shows that the structure of MOF is rigid enough to provide sharp molecular sieving, although the effective pore size is not exact the same 
as that is calculated from the crystallographic structure data. One reason is probably because ZIF8 structure is based on a stable connection. Recently, MOF structures based on stable triangle connections were also shown to give excellent molecular sieving effect [51]. Propylene is the second largest gas product after ethylene. It is mainly produced from the fluid catalytic cracking process which yields about $70 \%$ propylene and $30 \%$ propane. The separation is currently achieved by distillation. Because of the close boiling points of these two gases, the distillation process requires more than 200 separation trays, making it one of the most energy and capital intensive processes in petrochemical industry. The superstructure of the membrane-distillation hybrid system is shown in Figure 2a, which covers different situations including the pure distillation process, the membrane-distillation hybrid process, and the pure single-stage membrane process. The simulated energy consumption of the membrane-distillation hybrid system at different membrane selectivity and pressure ratio is shown in Figure $2 \mathrm{~b}$. It shows that a membrane with selectivity higher than 10 can help reduce the energy consumption from distillation. A membrane with selectivity of 30 and 100 can reduce $40 \%$ and $80 \%$ energy, respectively. Figure 2 c shows the separation performance of ZIF-8 membranes in comparison with other types of membranes. Clearly, the performance of ZIF-8 membranes achieved so far is not only much better than any other types of membranes, but also high enough to achieve up to $80 \%$ energy saving as predicted from modelling. However, most of the permeation tests in laboratory are carried out at room temperature, with 50:50 feed composition, and under no pressure drop. In real applications, the temperature is around $50^{\circ} \mathrm{C}$, feed contains $70 \%$ ethylene, and the pressure is about 18 bars [52]. As such, how to maintain the high membrane performance at real conditions will be a crucial step for commercialization. 

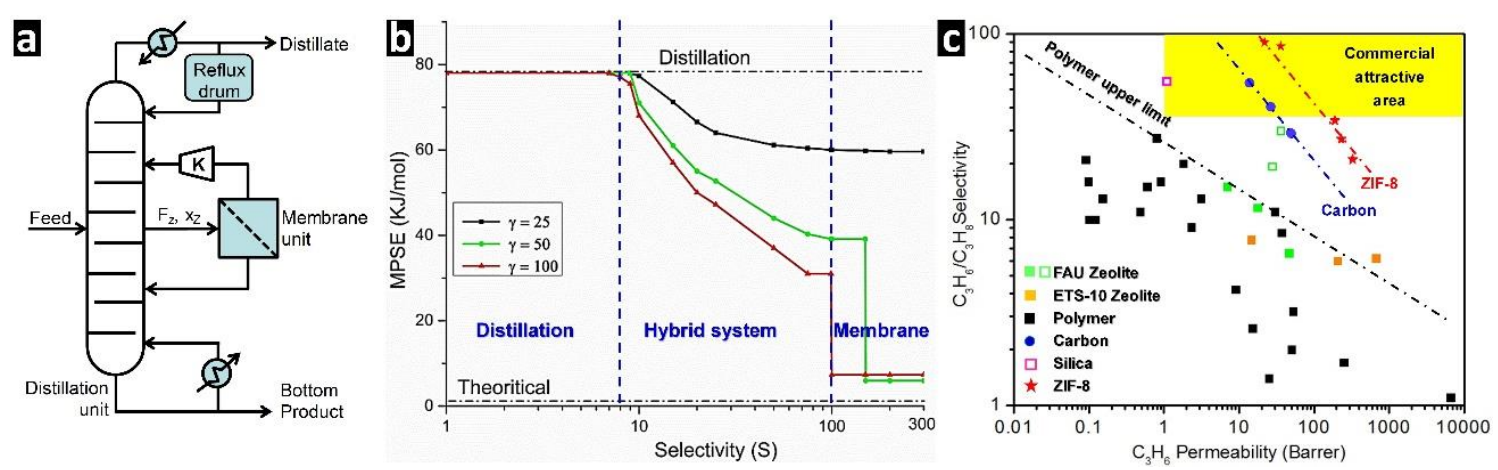

Figure 2: (a) the superstructure of the membrane-distillation system [ $2^{\circ}$ ]; (b) The simulated energy consumption for the separation of propylene/propane at different membrane selectivity and pressure ratio $\gamma\left[2^{\circ}\right]$. A sharp drop in energy in the third stage indicates that the hybrid system is downgraded into a single-stage membrane process. (c) Performance comparison of ZIF-8 membranes to other membrane systems for the separation of propylene/propane [46*0].

\section{Membrane stability}

From the bond strength calculations, ZIF-8 is one of the most stable MOF structures [5]. This is also confirmed by TEM studies where high resolution TEM images of the ZIF-8 structure can be

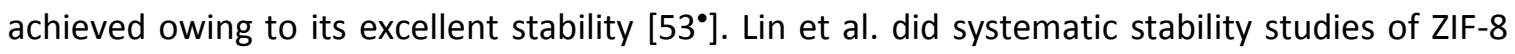
membranes $\left[54^{\bullet}, 55-56\right]$. Below is the summary of their main conclusions.

- ZIF-8 membrane can maintain structural integrity up to $150^{\circ} \mathrm{C}$.

- ZIF-8 membrane is not stable in liquid. The membrane is damaged in less than 10 hours exposure to water during a pervaporation test. The membrane is less stable than powder because it contains more defects. We also observed that ZIF- 8 membrane is not stable in organic solvents (unpublished data). In this study, a ZIF-8 membrane was used to separate the mixture of ethanol and propanol. The membrane showed excellent selectivity initially ( 35), but disappeared after about one-month running. Lin et al. further found that the ZIF-8 membrane degradation in liquid is a dynamic dissolution process. By adding 
precursors to the liquid the dissolution process is retarded. Since most MOF and ZIF materials are based on the same coordination chemistry, this dissolution mechanism should apply to all MOF membranes.

- For gas separation, the membrane is much more stable. In ambient conditions the membrane permeance drops only $25 \%$ after 35 days off-steam testing and the selectivity remained almost the same.

Jeong et al. compared the stability of ZIF-8 membranes prepared by two methods, one by counterdiffusion using methanol as solvent (CD-ZIF-8), and the other by microwave assisted seeding followed by secondary growth in aqueous solution (MW-ZIF-8). They found MW-ZIF-8 is more stable than CD-ZIF-8 and the reason is because MW-ZIF-8 contains less defects [57]. Lin et al.[58] and Yang et al.[59] found that the membrane stability can be improved by surface modification with hydrophobic ligands. However, the membrane stability under real applications still remains an open question.

\section{Mechanical strength and process reproducibility}

Many process reproducibility issues are related to the poor mechanical strength of ZIF-8. The structure is found very fragile and special care is required during solvent exchange. Direct removal of big solvent such as $\mathrm{N}, \mathrm{N}$-dimethyl formamide (DMF) or strong binding solvent such as water will cause structural collapse. Methanol has a small size and weak interaction with ZIF-8 structure and hence is commonly used as an exchanging solvent. The exchange rate is also an important factor. Using mixed solvent or saturated methanol vapor can slow down the exchange rate and therefore lead to better membrane quality [49].

The mechanical strength under pressure is another big challenge. Pan et al. found that the propylene/propane selectivity decreases from 61 to 14 when TMP increases from 0 to 3 bars [20]. 
The reason seems related to structure flexibility instead of physical damage since the selectivity can be largely restored when TMP is recovered. Nair et al. observed a similar trend in their studies $[31,43]$.

A good progress was made by Pan et al. recently using coating as an effective approach to eliminate defects [60*0. They applied a PDMS coating on defective ZIF-8 membranes and found that all membranes after treatment showed very high propylene to propane selectivity. Very interesting, the membrane selectivity after treatment increases with pressure. When TMP increased from 0 to 6 bars, the propylene/propane selectivity increased from 93 to 105, while when TMP released back to zero, the separation factor returned back to 95, very close to the initial value. The intrinsic-hydrophobic PDMS coating also significantly improved the hydrolytic stability of the membrane. The membrane exhibited excellent long-term stability even using humidified-state feed gases. Coating has been widely used in polymer membrane preparation as a remedy approach. Hence, this finding represents an important progress towards commercialization.

One should also note that the effect of structure flexibility is not always negative. Very recently, Caro et al. used an electrical field to distort the ZIF-8 structure, which can fine tune the gas separation performance [61].

\section{Perspectives of ZIF-8 membranes in industrial applications}

There are thus far no major industrial gas separation processes using crystalline porous membranes. The future of MOF membranes will largely depends on whether it can find an industrial application.

All three synthetic methods are able to fabricate high quality ZIF-8 membranes on hollow fiber supports. A number of post-treatment methods have been developed to further improve the 
membrane separation performance [62] or hydrothermal stability $[58,59]$. The CD method with cycling precursor streams from both sides indicates that a continuous synthesis process can be designed. Hence, the membrane productivity may not be a major issue. Similar to polymer membranes, coating is likely to be an essential step in membrane fabrication to eliminate defects. Considering that the $C D$ method can also repair defects in situ, the membrane reproducibility issue may not be as bad as it appears in the existing literature. Polymer is likely to be used as a support material because of its cheap price. Water used as solvent has apparent advantages in terms of cost and environmental impact compared to toxic and flammable organic solvents. Hence, even though the membrane cost may not be as cheap as those of polymer membranes, ZIF-8 membranes are still much more economical than other inorganic membranes.

The application of MOF membranes in liquid separations has been recently reviewed [63]. However, for ZIF-8 membrane it is unlikely suitable for liquid separations because of its poor stability in both water and organic solvents. However, for gas separation the long-term membrane stability under ambient conditions seems good. Because the effective pore size of ZIF-8 is about $0.07 \mathrm{~nm}$ larger than its crystallographic pore size, which makes it less selective and thus less competitive for $\mathrm{H}_{2}$ and $\mathrm{CO}_{2}$ separations. The membrane showed sharp molecular sieving for $\mathrm{C}_{2} / \mathrm{C}_{3}$ and propylene/propane mixtures, both are highly valuable systems in petrochemical industry. The selectivity is high enough to bring up to $80 \%$ energy reduction from the existing distillation process. Such a huge energy benefit will serve as a strong driving force to promote its commercialization. However, the membrane stability under real streams remains unknown. All the membrane permeation tests are carried out under conditions far away from the real separation conditions. The main challenge under real conditions is probably the mechanic strength under high pressure. The highest TMP tested so far is about 6 bars, while the pressure in real conditions is around 18 bars. How to maintain the high separation performance under such high pressure is unknown. 
There are almost no studies on other membrane challenges such as fouling and concentration polarization. Since the highest permeance of propylene reported so far is less than $100 \mathrm{GPU}$,

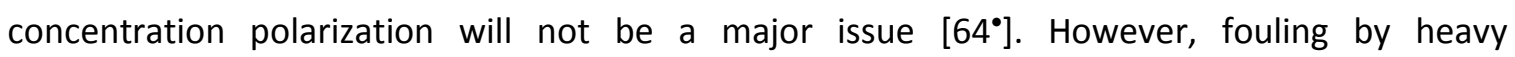
hydrocarbons can be another big challenge in real applications.

\section{Conclusions}

ZIF-8 as an eminent example of MOF membranes have shown very promising performance for the separation of propylene/propane. The current membrane synthetic methods are capable to fabricate ZIF-8 membrane modules suitable for industrial applications at competitive cost. Although there are still a number challenges in membrane stability and process reproducibility, there is no technically unsolvable barriers to stop it from commercialization. The high value and

large scale of the propylene/propane system and the potential energy benefit that can be achieved by a ZIF-8 membrane compared to the current distillation process will serve as a strong driving force to promote its commercialization. The successful implement of ZIF- 8 membrane in industry will greatly benefit the development of MOF membranes.

\section{Acknowledgements}

ZP Lai acknowledges the support of KAUST baseline fund BAS/1/1375 and CCF fund FCC/1/197227-01.

\section{References and recommended reading}

Papers of particular interest, published within the period of review, have been highlighted as:

- of special interest

$\bullet \bullet$ of outstanding interest 
1. Alshehri A, Lai ZP: Attainability and minimum energy of multiple-stage cascade membrane systems. J. Membr. Sci. 2015, 495:284-293.

2. Alshehri A, Lai ZP: Attainability and minimum energy of single-stage membrane and membrane/distillation hybrid processes. J. Membr. Sci. 2014, 472:272-280.

- A modelling study shows that membrane selectivity and pressure ratio are the two important design parameters.

3. Huang XC, Lin YY, Zhang JP, Chen XM: Ligand-directed strategy for zeolite-type metal-organic frameworks: zinc(ii) imidazolates with unusual zeolitic topologies. Angew. Chem. Int. Ed. 2006, 45:1557-1559.

•• The first report of ZIF-8 synthesis.

4. Park SP, Ni Z, Cote AP, Choi JY, Huang R, Uribe-Romo FJ, Chae HK, O'Keeffe M, Yaghi OM: Exceptional chemical and thermal stability of zeolitic imidazolate frameworks. Proc. Nat/. Acad. Sci. 2006, 103:10186-10191.

5. Low JJ, Benin Al, Jakubczak P, Abrahamina JF, Faheem SA, Willis RR: Virtual high throughput screening confirmed experimentally: porous coordination polymer hydration. J. Am. Chem. Soc. 2009, 131:15834-15842.

6. Cravillon J, Munzer S, Lohmeier SJ, Feldhoff A, Huber K, Wiebcke M: Rapid room-temperature synthesis and characterization of nanocrystals of a prototypical zeolitic imidazolate framework. Chem. Mater. 2009, 21:1410-1412.

-• A good progress in ZIF-8 synthesis which makes the synthesis much faster and easier.

7. Pan YC, Liu YY, Zeng GF, Zhao L, Lai ZP: Rapid synthesis of zeolitic imidazolate framework-8 (ZIF-8) nanocrystals in an aqueous system. Chem. Commun. 2011, 47:2071-2073.

-• The first report of ZIF-8 synthesis in aqueous solution. 
8. Bux H, Liang F, Li Y, Cravillon J, Wiebcke M, Caro J: Zeolitic imidazolate framework membrane with molecular sieving properties by microwave-assisted solvothermal synthesis. J. Am. Chem. Soc. 2009, 131:16000-16001.

-• The first report of ZIF-8 membrane synthesis.

9. Caro J: Are MOF membranes better in gas separation than those made of zeolites? Curr. Opin. Chem. Eng. 2011, 1:77-83.

10. Caro J: Are MOF membranes betterthan those made of zeolites? In Procedia Engineering: 2012:1-2.

11. Keskin S, van Heest TM, Sholl DS: Can metal-organic framework materials play a useful role in large-scale carbon dioxide separations? ChemSusChem 2010, 3:879-891.

12. Shekhah O, Liu J, Fischer RA, Wöll C: MOF thin films: Existing and future applications. Chem. Soc. Rev. 2011, 40:1081-1106.

13. Qiu S, Xue M, Zhu G: Metal-organic framework membranes: From synthesis to separation application. Chem. Soc. Rev. 2014, 43:6116-6140.

14. Rangnekar N, Mittal N, Elyassi B, Caro J, Tsapatsis M: Zeolite membranes - a review and comparison with MOFs. Chem. Soc. Rev. 2015, 44:7128-7154.

-• A very good reivew to combine the progresses in both zeolite and MOF membranes.

15. Liu Y, Ban Y, Yang W: Microstructural engineering and architectural design of metal-organic framework membranes. Adv. Mater. 2017, 29:1606949.

16. Zhu Y, Liu Q, Caro J, Huang A: Highly hydrogen-permselective zeolitic imidazolate framework ZIF-8 membranes prepared on coarse and macroporous tubes through repeated synthesis. Sep. Purif. Technol. 2015, 146:68-74. 
17. Tanaka S, Okubo K, Kida K, Sugita M, Takewaki T: Grain size control of ZIF-8 membranes by seeding-free aqueous synthesis and their performances in propylene/propane separation. J. Membr. Sci. 2017, 544:306-311.

18. Huang A, Liu Q, Wang N, Caro J: Highly hydrogen permselective ZIF-8 membranes supported on polydopamine functionalized macroporous stainless-steel-nets. J. Mater. Chem. A 2014, 2:8246-8251.

19. Khaletskaya K, Turner S, Tu M, Wannapaiboon S, Schneemann A, Meyer R, Ludwig A, Van Tendeloo G, Fischer RA: Self-directed localization of ZIF-8 thin film formation by conversion of ZnO nanolayers. Adv. Funct. Mater. 2014, 24:4804-4811.

20. Yu J, Pan Y, Wang C, Lai ZP: ZIF-8 membranes with improved reproducibility fabricated from sputter-coated ZnO/alumina supports. Chem. Eng. Sci. 2016, 141:119-124.

21. Zhang X, Liu Y, Kong L, Liu H, Qiu J, Han W, Weng LT, Yeung KL, Zhu W: A simple and scalable method for preparing low-defect ZIF-8 tubular membranes. J. Mater. Chem. A 2013, 1:10635-10638.

22. Wang X, Sun M, Meng B, Tan X, Liu J, Wang S, Liu S: Formation of continuous and highly permeable ZIF-8 membranes on porous alumina and zinc oxide hollow fibers. Chem. Commun. 2016, 52:13448-13451.

23. Barankova E, Tan X, Villalobos LF, Litwiller E, Peinemann KV: A metal chelating porous polymeric support: the missing link for a defect-free metal-organic framework composite membrane. Angew. Chem. Int. Ed. 2017, 56:2965-2968.

24. Liu YY, Zeng GF, Pan YC, Lai ZP: Synthesis of highly c-oriented ZIF-69 membranes by secondary growth and their gas permeation properties. J. Membr. Sci. 2011, 379:46-51.

25. Tao K, Kong C, Chen L: High performance ZIF-8 molecular sieve membrane on hollow ceramic fiber via crystallizing-rubbing seed deposition. Chem. Eng. J. 2013, 220:1-5. 
26. Kong L, Zhang X, Liu H, Qiu J: Synthesis of a highly stable ZIF-8 membrane on a macroporous ceramic tube by manual-rubbing $\mathrm{ZnO}$ deposition as a multifunctional layer. J. Membr. Sci. 2015, 490:354-363.

27. Kwon HT, Jeong HK: Highly propylene-selective supported zeolite-imidazolate framework (ZIF-8) membranes synthesized by rapid microwave-assisted seeding and secondary growth. Chem. Commun. 2013, 49:3854-3856.

28. Brown AJ, Brunelli NA, Eum K, Rashidi F, Johnson JR, Koros WJ, Jones CW, Nair S: Interfacial microfluidic processing of metal-organic framework hollow fiber membranes. Science 2014, 345:72-75.

-• A good progress in the counter-diffusion method which indicates the potential to synthesize ZIF-8 membranes on polymeric supports through a continuous flow system.

29. Yao J, Dong D, Li D, He L, Xu G, Wang H: Contra-diffusion synthesis of ZIF-8 films on a polymer substrate. Chem. Commun. 2011, 47:2559-2561.

•• The first report of counter-diffusion method for ZIF-8 membrane synthesis.

30. Kwon HT, Jeong HK: In situ synthesis of thin zeolitic-imidazolate framework ZIF-8 membranes exhibiting exceptionally high propylene/propane separation. J. Am. Chem. Soc. 2013, 135:10763-10768.

-• This report shows that local defects can be repaired in situ by a modified counter-diffusion method.

31. Eum K, Rownaghi A, Choi D, Bhave RR, Jones CW, Nair S: Fluidic processing of highperformance ZIF-8 membranes on polymeric hollow fibers: mechanistic insights and microstructure control. Adv. Funct. Mater. 2016, 26:5011-5018. 
32. Kong L, Zhang X, Liu Y, Li S, Liu H, Qiu J, Yeung KL: In situ fabrication of high-permeance ZIF-8 tubular membranes in a continuous flow system. Mater. Chem. Phys. 2014, 148:10-16.

33. Huang K, Dong Z, Li Q, Jin W: Growth of a ZIF-8 membrane on the inner-surface of a ceramic hollow fiber via cycling precursors. Chem. Commun. 2013, 49:10326-10328.

34. Marti AM, Wickramanayake W, Dahe G, Sekizkardes A, Bank TL, Hopkinson DP, Venna SR: Continuous flow processing of ZIF-8 membranes on polymeric porous hollow fiber supports for $\mathrm{CO}_{2}$ capture. ACS Appl. Mater. Interfaces 2016, 9:5678-5682.

35. Patterson JP, Abellan P, Denny MS, Park C, Browning ND, Cohen SM, Evans JE, Gianneschi C: Observing the growth of metal-organic frameworks by in situ liquid cell transmission electron microscopy. J. Am. Chem. Soc. 2015, 137:7322-7328.

36. Shah MN, Gonzalez MA, McCarthy MC, Jeong HK: An unconventional rapid synthesis of high performance metal-organic framework membranes. Langmuir 2013, 29:7896-7902.

37. Centrone A, Yang Y, Speakman S, Bromberg L, Rutledge GC, Hatton TA: Growth of metalorganic frameworks on polymer surfaces. J. Am. Chem. Soc. 2010, 132:15687-15691.

- The first trial to prepare MOF layer on a polymer surface.

38. Ben T, Lu C, Pei C, Xu S, Qiu S: Polymer-supported and free-standing metal-organic framework membrane. Chem. Eur. J. 2012, 18:10250-10253.

39. Cacho-Bailo F, Seoane B, Téllez C, Coronas J: ZIF-8 continuous membrane on porous polysulfone for hydrogen separation. J. Membr. Sci. 2014, 464:119-126.

40. Li W, Meng Q, Li X, Zhang C, Fan Z, Zhang G: Non-activation ZnO array as a buffering layer to fabricate strongly adhesive metal-organic framework/PVDF hollow fiber membranes. Chem. Commun. 2014, 50:9711-9713. 
41. Cacho-Bailo F, Catalán-Aguirre S, Etxeberría-Benavides M, Karvan O, Sebastian V, Téllez C, Coronas J: Metal-organic framework membranes on the inner-side of a polymeric hollow fiber by microfluidic synthesis. J. Membr. Sci. 2015, 476:277-285.

42. Isaeva VI, Barkova MI, Kustov LM, Syrtsova DA, Efimova EA, Teplyakov VV: In situ synthesis of novel ZIF-8 membranes on polymeric and inorganic supports. J. Mater. Chem. A 2015, 3:7469-7476.

43. Eum K, Ma C, Rownaghi A, Jones CW, Nair S: ZIF-8 membranes via interfacial microfluidic processing in polymeric hollow fibers: efficient propylene separation at elevated pressures. ACS Appl. Mater. Interfaces 2016, 8:25337-25342.

44. Hou J, Sutrisna PD, Zhang Y, Chen V: Formation of ultrathin, continuous metal-organic framework membranes on flexible polymer substrates. Angew. Chem. Int. Ed. 2016, 55:3947-3951.

- This report indicates that ZIF-8 membranes on polymer supports can tolerate certain degree of bending and elogation with no loss in separation performance.

45. Pan YC, Lai ZP: Sharp separation of $C_{2} / C_{3}$ hydrocarbon mixtures by zeolitic imidazolate framework-8 (ZIF-8) membranes synthesized in aqueous solutions. Chem. Commun. 2011, 47:10275-10277.

-• The first report shows sharp molecular sieving for $C_{2} / C_{3}$ mixtures on ZIF-8 membranes.

46. Pan YC, Li T, Lestari G, Lai ZP: Effective separation of propylene/propane binary mixtures by ZIF-8 membranes. J. Membr. Sci. 2012, 390-391:93-98.

-• The first report of propylene/propane separation on ZIF-8 membranes.

47. Chen NY, Degnan TF, Smith CM: Molecular Transport and Reaction in Zeolites: Design and Application of Shape Selective Catalysts. New York: Wiley-VCH; 1994. 
48. Fairen-Jimenez D, Moggach SA, Wharmby MT, Wright PA, Parsons S, Duren T: Opening the gate: framework flexibility in ZIF-8 explored by experiments and simulations. J. Am. Chem. Soc. 2011, 133:8900-8902.

49. Pan Y, Liu W, Zhao Y, Wang C, Lai ZP: Improved ZIF-8 membrane: Effect of activation procedure and determination of diffusivities of light hydrocarbons. J. Membr. Sci. 2015, 493:88-96.

50. Li K, Olson DH, Seidel J, Emge TJ, Gong H, Zeng H, Li J: Zeolitic imidazolate frameworks for kinetic separation of propane and propene. J. Am. Chem. Soc. 2009, 131:10368-10369.

51. Xue DX, Belmabkhout Y, Shekhah O, Jiang H, Adil K, Cairns AJ, Eddaoudi M: Tunable rare earth fcu-MOF platform: access to adsorption kinetics driven gas/vapor separations via pore size contraction. J. Am. Chem. Soc. 2015, 137:5034-5040.

52. Gokhale V, Hurowitz S, Riggs JB: A comparison of advanced distillation control techniques for a propylene/propane splitter. Ind. Eng. Chem. Res. 1995, 34:4413-4419.

53. Zhu YH, Ciston J, Zheng B, Miao XH, Czarnik C, Pan YC, Sougrat R, Lai ZP, Hsiung C, Yao KX, et al.: Atomic-resolution imaging of a metal-organic framework: unravelling surface and interfacial structures. Nat. Mater. 2017, 16:532-536.

- $\quad$ The first high resolution TEM image of ZIF-8 structure.

54. Zhang H, Liu D, Yao Y, Zhang B, Lin YS: Stability of ZIF-8 membranes and crystalline powders in water at room temperature. J. Membr. Sci. 2015, 485:103-111.

-• A comprehensive study on the stability of ZIF-8 membranes.

55. James JB, Lin YS: Thermal stability of ZIF-8 membranes for gas separations. J. Membr. Sci. 2017, 532:9-19. 
56. Liu D, Ma X, Xi H, Lin YS: Gas transport properties and propylene/propane separation characteristics of ZIF-8 membranes. J. Membr. Sci. 2014, 451:85-93.

57. Lee MJ, Kwon HT, Jeong HK: Defect-dependent stability of highly propylene-selective zeolitic-imidazolate framework ZIF-8 membranes. J. Membr. Sci. 2017, 529:105-113.

58. Zhang H, James J, Zhao M, Yao Y, Zhang Y, Zhang B, Lin YS: Improving hydrostability of ZIF-8 membranes via surface ligand exchange. J. Membr. Sci. 2017, 532:1-8.

59. Liu X, Li Y, Ban Y, Peng Y, Jin H, Bux H, Xu L, Caro J, Yang W: Improvement of hydrothermal stability of zeolitic imidazolate frameworks. Chem. Commun. 2013, 49:9140-9142.

60. Sheng L, Wang C, Yang F, Xiang L, Huang X, Yu J, Zhang L, Pan Y, Li Y: Enhanced $C_{3} \mathbf{H}_{6} / \mathbf{C}_{3} \mathrm{H}_{8}$ separation performance on MOF membranes through blocking defects and hindering framework flexibility by silicone rubber coating. Chem. Commun. 2017, 53:7760-7763.

- A very good progress shows that coating as a post-treatment can be an efficient approach to eliminate defects, to enhance the membrane mechanical strenght under pressure, as well as to improve the membrane hydrothermal stability.

61. Knebel A, Geppert B, Volgmann K, Kolokolov DI, Stepanov AG, Twiefel J, Heitjans P, Volkmer D, Caro J: Defibrillation of soft porous metal-organic frameworks with electric field. Science 2017, 358:347-351.

62. Lee MJ, Kwon HT, Jeong HK: High-flux zeolitic imidazolate framework membranes for propylene/propane separation by postsynthetic linker exchange. Angew. Chem. Int. Ed. 2018, 57:156-161.

63. Li X, Liu J, Wang J, Gascon J, Li J, Bruggen BV: Metal-organic frameworks based membranes for liquid separation. Chem. Soc. Rev. 2017, 46:7124-7144.

64. He G, Mi Y, Yue PL, Chen G: Theoretical study on concentration polarization in gas separation membrane processes. J. Membr. Sci. 1999, 153:243-258. 
- A simple mathematic model is developed to predict concentration polarization in gas separation membrane processes. 